\title{
Evolution of Synapses and Neurotransmitter Systems: The Divide-and-Conquer Model for Early Neural Cell-Type Evolution
}

\author{
Pawel Burkhardt ${ }^{1}$ and Gáspár Jékely ${ }^{2}$ \\ ${ }^{1}$ Sars International Centre for Marine Molecular Biology, University of Bergen, Norway \\ ${ }^{2}$ Living Systems Institute, University of Exeter, Stocker Road, Exeter, UK \\ Pawel.Burkhardt@uib.no, g.jekely@exeter.ac.uk
}

\begin{abstract}
Nervous systems evolved around 560 million years ago to coordinate and empower animal bodies. Ctenophores - one of the earliest-branching lineages - are thought to share few neuronal genes with bilaterians and may have evolved neurons convergently. Here we review our current understanding of the evolution of neuronal molecules in non-bilaterians. We also reanalyse single-cell sequencing data in light of new cell-cluster identities from a ctenophore and uncover evidence supporting the homology of one ctenophore neuron-type with neurons in Bilateria. The specific coexpression of the presynaptic proteins Unc13 and RIM with voltage-gated channels, neuropeptides and homeobox genes pinpoint a spiking sensory-peptidergic cell in the ctenophore mouth. Similar Unc13-RIM neurons may have been present in the first eumetazoans to rise to dominance only in stem Bilateria. We hypothesize that the Unc13-RIM lineage ancestrally innervated the mouth and conquered other parts of the body with the rise of macrophagy and predation during the Cambrian explosion.
\end{abstract}

Keywords: choanoflagellates, sponges, ctenophores, placozoans, cnidarians, synapse, neuron, evolution, neuropeptides

\section{Phylogenetic overview - set the stage for nervous system origins}

Nervous systems probably first appeared on our planet shortly before 560 million years ago. The earliest indirect evidence for the presence of a nervous system are Precambrian trace fossils left by the earliest bilaterians ca. $558 \mathrm{Ma}$ [1]. By this time, the major groups of animals, including the bilaterians, cnidarians, placozoans, ctenophores and sponges had likely already diverged.

With the exception of sponges and placozoans (and some highly reduced parasites), all animals have a nervous system. Animal nervous systems are morphologically, functionally and molecularly highly diverse and disparate. No universal neuronal markers have been found and the earliest transitions leading to nervous systems remain shrouded in mystery. It is clear that any attempt to reconstruct nervous system evolution requires a broad sampling across animal phylogeny. Non-bilaterian animals deserve special attention in such endeavours. These include the neuron-less sponges and placozoans (disk-shaped millimetre-sized ciliated marine animals) and the neuronal cnidarians (jellyfish, corals, sea anemones and relatives) and ctenophores or comb jellies (gelatinous marine invertebrates). In recent years, we have seen a revival of interest in these animals with an initial focus on genomics and phylogeny and more recently also on molecular function and neurophysiology.

Reconstructing ancestral states and transitions in nervous system evolution requires a robust phylogenetic framework. After a lot of flux, a reliable phylogeny for the major groups of non-bilaterian animals begins to emerge. Sponges seem to re-establish their position as the first animal group to branch from the rest of the animals. Their placement can only be explained by real phylogenetic 
signal, while the other contestants for this key position, the ctenophores, were often pushed as first due to systematic error [2]. Ctenophores now seem to be the second branch off the main animal tree, from the perspective of the bilaterians, followed by cnidarians and their likely sisters, the placozoans [3]. More stable has been the position of the choanoflagellates, our closest protist relatives. These single-celled or colonial organisms, characterised by a cilium surrounded by a microvillar collar, also have special relevance to understanding the evolutionary assembly of neuronal pathways $[4,5]$.

The timeline of animal origins has also been controversial with conflicting dates given by molecular clocks, fossil biomarkers and morphological fossils. A congruence of dates is now emerging, with molecular clock estimates becoming less credible [6,7] and with increasing ambiguity surrounding Ediacaran putative sponge fossils (e.g. [8]) and biomarkers [9-12]. At the same time, paleontologists increasingly succeed in placing fossils in a credible phylogenetic context providing minimum estimates for the appearance and ancestral morphology of the major animal lineages (e.g., [12,13]). Some Ediacaran fossils of previously enigmatic affinity (Dickinsonia and its relatives) have also been confirmed as animals based on steroid biomarker evidence, providing an important piece to the puzzle [14]. Our best understanding of the timeline and phylogeny of early animal diversification is summarised in Figure 1. This framework will form the basis of our discussion in this review.

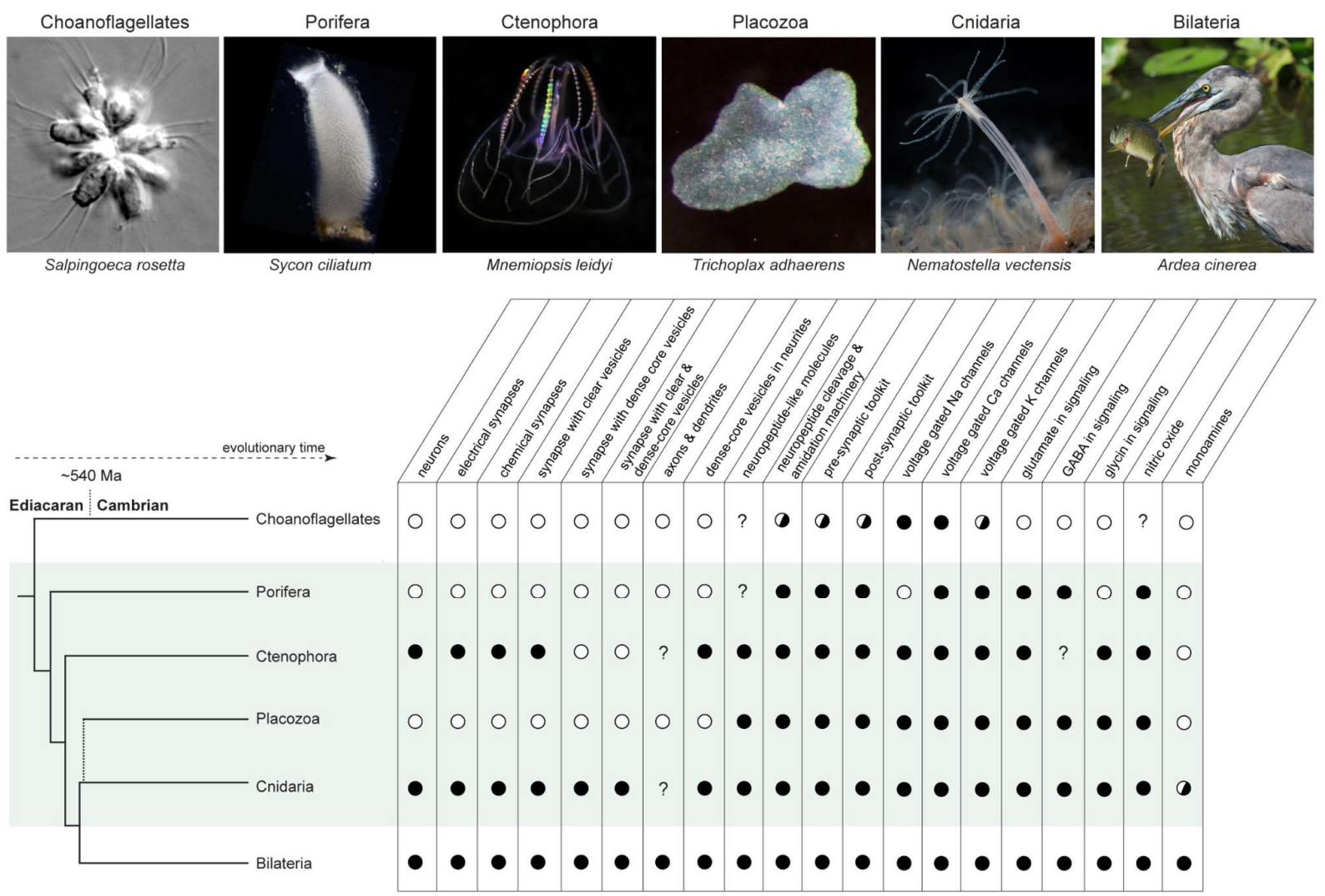

Figure 1: Animal phylogeny and the distribution of neuronal characters. (A) Representatives of the main animal lineages and their closest protist relatives. (B) Simplified animal phylogeny and character distribution across the main animal lineages and choanoflagellates. Phylogeny adapted from [2].

Defining a neuron - the hunt for elusive universal neuronal markers 
A nervous system consists of excitable cells with long projections that connect to each other and other cells by synapses. Excitability is the ability of the cells to produce highly non-linear ionic responses that can propagate in the cells along the processes and spread to other cells at synapses. All of these characteristics of nervous systems are supported by dedicated cellular components and processes often specific to neuronal cells.

The neuronal components can include specialised sensory complexes in sensory neurons, neurotransmitter and neuromodulator synthesis and transport systems, transmitter receptors, various ion channels, or pre- and postsynaptic complexes. It is therefore not an unreasonable expectation that by using these molecules as markers, we should be able to identify neurons in any nervous system. This view has become less and less tenable over the last decade. Molecular studies in nonbilaterian animals instead suggested that the further away we move from the Bilateria, the more elusive the molecular signatures of neurons become [15]. This applies to neuronal specification molecules, neurotransmitters and their processing machinery, neuropeptides and their receptors, and synaptic proteins. Key molecular components of bilaterian nerves are often not expressed in neurons of non-bilaterians or not present in ctenophores [16]. The history of neuronally-expressed gene families is often difficult to reconstruct and in many cases these are not characterised by one-to-one but one-to-many or many-to-many orthology relationships. These patterns indicate extensive parallel evolution in neuronal systems and make specific functional assignments difficult. Single-cell sequencing studies also suggest a striking heterogeneity of gene expression in ctenophore neurons [17].

In other instances, the molecular players may be well conserved, but their expression diverges and while they can be robust neuron markers in bilaterians, they are not in non-bilaterians.

These patterns led to the suggestion that the nervous system of ctenophores and cnidarians+bilaterians evolved convergently [18]. Here we revisit this influential hypothesis in light of new evidence.

\section{What were the first neurotransmitters?}

The evolution of neurotransmitter systems has recently been extensively reviewed [19]. In this section, we give a brief overview of recent findings relevant to reconstruct the ancestry of neural systems.

The small neurotransmitters and monoamines familiar from bilaterians are only partially present in non-bilaterians. Only glycine and glutamate are generally involved in signalling in non-bilaterians and are the best candidates as the first neurotransmitters at the origin of nervous systems. In placozoans, glycine is the most abundant transmitter and exogenous glycine induces tissue contractility and changes ciliary activity [20]. In ctenophores, glutamate and glycine are the only small transmitters identified. Muscle cells in Pleurobrachia bachei generate action potentials upon the application of both D- and L-glutamate.

Ctenophore genomes encode over a dozen homologs of bilaterian ionotropic glutamate receptors (iGluRs). Biochemical and electrophysiological analysis of several of these from Mnemiopsis leidyi and $P$. bachei revealed that many are activated by glycine $[21,22]$. Other iGluRs are activated by glutamate with glycine acting as an antagonist. The presence of these receptors together with the physiological effects of glutamate and glycine makes glutamate and glycine strong candidates for neurotransmitters in ctenophores. The iGluR family has a more ancient origin and is also present in some sponges [23]. Broader sampling in choanoflagellates also revealed the sporadic presence of iGluR sequences in some species, indicating that the family predates animals (PB and Tarja Hoffmeyer, unpublished).

The evidence is more fragmentary for GABA. GABA is present in placozoans [20], cnidarians (in the jellyfish Aequorea, GABA is a dominant analyte) and sponges. In sponges, GABA can induce 
contractions or block the action of glutamate, depending on the species [24,25]. However, the application of GABA did not seem to have an effect on muscle cells in Pleurobrachia bachei [16] and ctenophores seem to have lost most genes of the GABA pathway [26]. No specific GABA receptors have been cloned and characterised from any non-bilaterian, but candidate receptors exist in sponges [26]. Acetylcholine as a transmitter is likely a bilaterian innovation as non-bilaterians lack its synthesis, transport and degradation pathways [19].

The monoamine transmitters known from bilaterian neurons (dopamine, serotonin, octopamine, tyramine, noradrenaline) also likely appeared only in stem Bilateria. No monoamine receptors, full synthesis pathway or vesicular transporters have been found in non-bilaterian genomes [19,26]. In placozoans and ctenophores, capillary electrophoresis failed to detect serotonin, dopamine, or octopamine [20]. The G-protein coupled receptor (GPCR) families for octopamine, tyramine and noradrenaline diversified in stem bilaterians with no evidence for direct non-bilaterian orthologs [27]. Although chemical analyses identified both serotonin and dopamine for example in the hydrozoan Hydra magnipapillata [28] or the sea anemone Aiptasia pallida [29], there is no evidence that these molecules function as neurotransmitters or are produced by neurons. Only rarely, such as in the anthozoan Renilla koellikeri, was serotonin localised to neurons by immunohistochemistry [30].

Melatonin may be the only monoamine hormone shared between cnidarians and bilaterians. It was detected in the sea anemones Actinia equina [31] and Nematostella vectensis [32] where its levels showed diel oscillations, consistent with a role in circadian physiology. Intriguingly, the jellyfish Cassiopea has a sleep-like state but the involvement of melatonin in this state is unclear [33]. Cnidarians and also placozoans have melatonin receptor orthologs [34], but these have not been characterised experimentally.

There is also the intriguing possibility that other, non-canonical monoamines may be deployed in neuronal signalling outside the Bilateria. This is suggested by the large number of orphan monoamineGPCR-like sequences in Cnidaria (GJ and Luis A Yañez-Guerra, unpublished).

Other non-conventional transmitters also occur in some non-bilaterians. In the jellyfish Cyanea capillata, taurine is a candidate neurotransmitter as it is present in the motor nerve net [35] and together with beta-alanine - can depolarise neurons [36]. Furthermore, the $D$ and $L$ enantiomers of both glutamate and aspartate in non-bilaterians deserve more attention, with D-glutamate having potentially unique transmitter roles in ctenophores [37].

\section{Paracrine signalling molecules}

Paracrine signalling through the non-synaptic release of small molecules is ubiquitous in animals. This mode of signalling may represent the first form of intercellular communication that allowed multicellular animals to coordinate tissue activity (e.g. contractions, secretion, ciliary movement) [37]. A widespread and ancient paracrine signalling molecule is nitric oxide (NO), produced by the enzyme nitric oxide synthase (NOS) [19]. NO can influence smooth muscle contraction, neuronal and developmental signalling. Placozoans have three NOS genes expressed in different subpopulations of cells close to the edge regions and capillary electrophoresis supports the presence of active NOS enzymes [38]. In ctenophores, only highly derived NOS enzymes have been found, and it is unclear whether these animals have functional NO signalling [38]. In the demosponge Spongilla lacustris, NOS is expressed in pinacocytes - cells that line the canal system - and exogenous NO induces contractions in cells of the incurrent canals [39].

A functionally and structurally more diverse class of paracrine signalling molecules are secreted neuropeptide-like molecules. Such peptides are produced from prepropeptides that undergo proteolytic cleavage and modifications in the secretory pathway. The mature peptides are released 
from dense core vesicles and can influence behaviour and development across eumetazoans [4043].

Signalling peptides were already present in the eumetazoan common ancestor [37] and potentially before. They are abundant in ctenophores [16,44], placozoans [16,42,45-47], cnidarians [48,49] and bilaterians [50-52] (Figure 2A). In sponges, no neuropeptide-like molecule has yet been identified but some may exist. This is suggested by the presence of prohormone convertases in sponges, enzymes that cleave prepropeptides, and of peptidylglycine $\alpha$-amidating monooxygenase (PAM), an enzyme required for peptide amidation (Figure 2B). PAM is an ancient enzyme also present across ciliated protists [53] and involved in the processing of amidated signalling peptides in the green alga Chlamydomonas [54]. In sponges, the substrates of PAM are unknown but sponge tissues contain dense core vesicles [55] [56] similar to peptidergic vesicles in eumetazoans nerves, suggesting the regulated release of peptides.

We know a lot less about peptide receptors. Although all non-bilaterian genomes contain many GPCRs - the most common peptide receptors - so far only one has been matched to a ligand, the receptor of the maturation inducing peptide $(\mathrm{MIH})$ in the jellyfish Clytia hemisphaerica [40]. In Hydra, RFamide-like peptides signal by ligand-gated ion channels [57]. These DEG/ENAC family channels are present in placozoans and also (greatly expanded) in ctenophores [16]. How widespread their involvement is in ionotropic peptidergic signalling is unknown. 


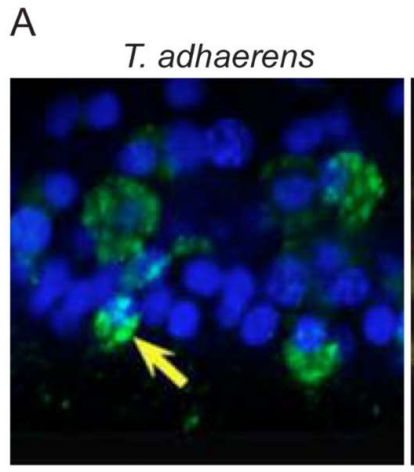

B
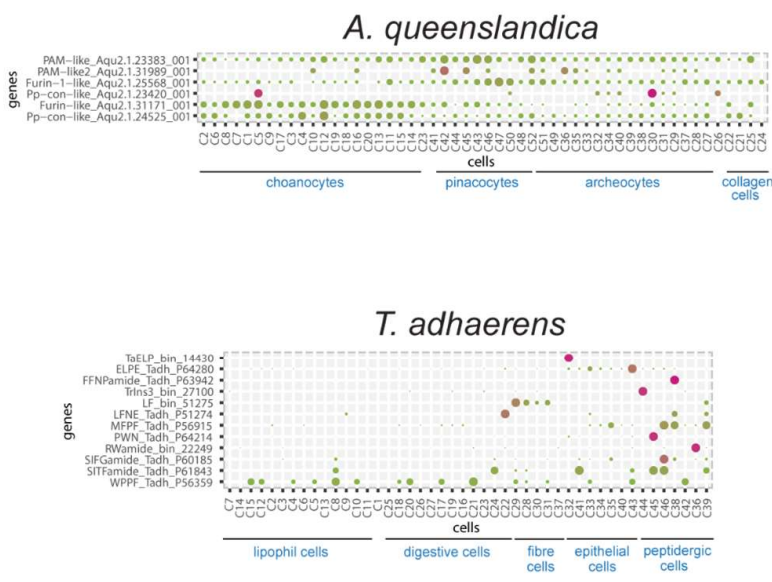

N. vectensis
N. vectensis

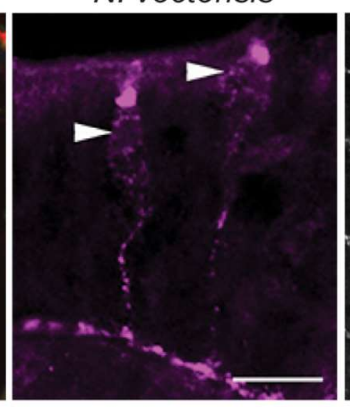

M. leidyi

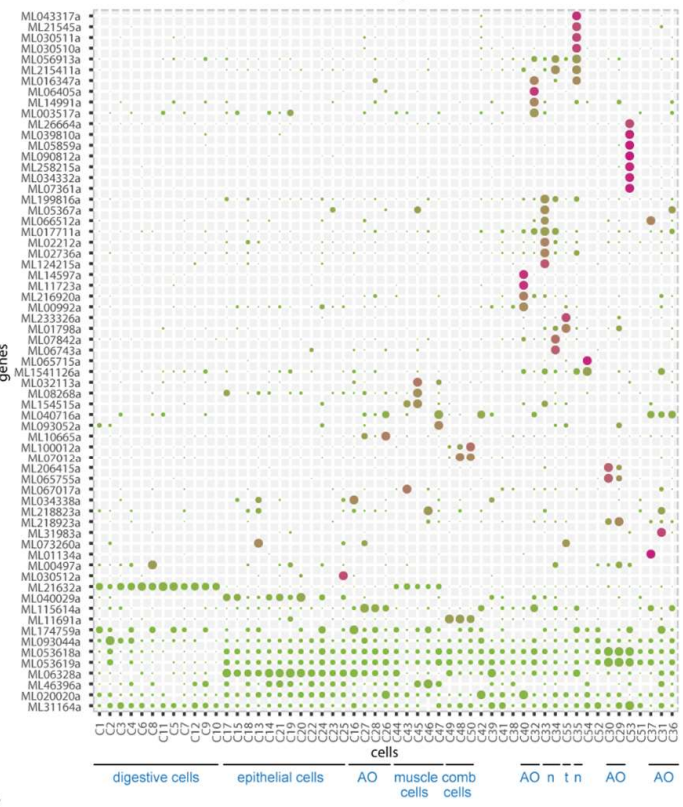

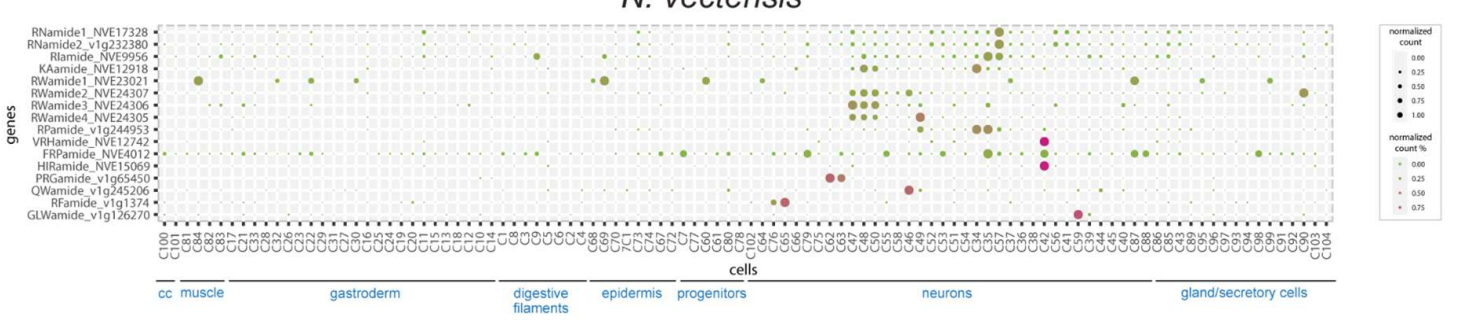

Figure 2: Neuropeptides in non-bilaterian animals. (A) Neuropeptide localization in the placozoan $T$. adhaerens, the ctenophore $M$. leidyi and the two cnidarians $N$. vectensis and $C$. hemisphaerica (modified from [43,44,58,59]) (B) Single-cell RNA sequencing data of prepropeptide processing enzyme genes across the annotated metacells in the sponge $A$. queenslandica, and prepropeptide genes in the placozoan $T$. adhaerens, the ctenophore $M$. leidyi and the cnidarian $N$. vectensis, based on [44,60,61]. Abbreviations: AO, aboral organ; $n$, neurons; pp-con, proprotein-convertase; $t$, tentacles, cc, cnidocytes

Tissue stainings and single-cell RNA sequencing approaches revealed the highly specific expression of most prepropeptides in all animals studied. To illustrate this, we plotted the expression of prepropeptide genes across cell clusters from the cnidarian Nematostella vectensis, the placozoan Trichoplax adhaerens, and the ctenophore Mnemiopsis leidyi based on the published datasets [60,61] 
(Figure 2B). In all three species, the prepropeptides show highly cell-type-specific expression, with the exception of some uncharacterised peptides in the ctenophore. The specific expression of these genes has recently allowed the identification of some of the cell clusters as neuronal-peptidergic cells in the ctenophore M. leidyi [44]. In the sponge Amphimedon queenslandica, PAM and secretory prohormone-convertase homologs - potentially involved in prepropeptide cleavage - are broadly expressed, suggesting the potentially broad deployment of signalling by amidated peptides.

These data suggest that secreted peptides were already produced and secreted by the first neurons and due to their highly specific expression and signalling through specific receptors contributed to these neurons' identity and signalling specificity. These signalling functions of peptides may have evolved before synapses.

\section{Synaptic proteins and synapse structure}

Synapses are one of the defining features of neurons and are contact points between neurons or neurons and effector cells, like muscle cells and secretory cells. Synapses evolved early in animals. Two different types can be distinguished, chemical and electrical. Both types of synapses have been identified in ctenophores and cnidarians at an ultrastructural level [62] [63] [64]. Here we focus on the presynaptic release machinery of chemical synapses and dense core vesicles.

Most, if not all of the molecular components of synapses (synaptic modules) evolved prior to the appearance of the first neurons and many proteins prior to the origin of the first animals [65][5]. For example, key proteins involved in the tightly regulated process of neurosecretion (e.g., SNAREs, Unc18, Unc13 and Complexin), active zone proteins (e.g., Rimbp) and core neurosecretory vesicle proteins (e.g., Synaptophysin and Synaptogyrin) are present in unicellular organisms closely related to animals. In addition, postsynaptic scaffolding proteins (e.g., PSD-95, Homer, Shank) and ionotropic glutamate receptors existed before the first animals. These findings highlight the difficulty to pinpoint specific "neuronal" proteins as molecular markers for synapses, as the ancestral functions of most of these proteins were likely non-neuronal. Below we highlight recent work characterizing the function of "synaptic" genes or proteins in non-bilaterian animals without and with synapses and neurons.

In an elegant study using whole body scRNAseq in the sponge Spongilla lacustris the authors found that sponge "presynaptic" genes are specifically expressed in the sensory contractile pinacocyte epithelium, and in a new cell type the authors refer to as neuroid cells [39]. The neuroid cell contains many different dense-core vesicle secretion genes and wraps around choanocytes, the feeding cells of sponges. Complementing this, "postsynaptic" genes are predominantly enriched in choanocytes. These experiments indicate a close interaction of two cell types expressing pre-and postsynaptic genes and thus may identify these two cell types as proto-neurons. The placozoan Trichoplax adhaerens has no recognizable neurons but possesses neuropeptide-like molecules that are expressed in elongated, vesicle-rich epithelial cells. Immunostaining experiments have revealed that these cells co-express synaptobrevin, complexin, and synaptophysin, proteins enriched in vertebrate synapses $[45,66]$. When compared to other animals with neurons, ctenophores possess a synaptic protein repertoire comparable to sponges, placozoans and cnidarians, although a few key molecules like Synaptotagmin1 and Neuroligins have not been identified in the genomes of $M$. leidyi and $P$. pileus [15]. However, localisation studies and functional characterization of putative synaptic proteins in ctenophores have yet to be performed. The recent identification of the neuropeptide repertoire in a ctenophore allowed for the annotation of many previously unknown ctenophore cell types and led to the identification of the molecular identity of the nerve net [44]. This work showed that the ctenophore nerve net expresses Ferlin and Segretagogin (putative synaptic vesicle markers) and multiple ion channels important for the generation of action potentials. It is so far unclear how activity is spreading through the whole ctenophore nerve net as neurons within the nerve net display a unique structure 
with neurites fused to each other (anastomoses) and no directionality (axons vs dendrites) can be distinguished [44].

Ultrastructural analysis of ctenophore neurons and many sensory cells revealed multiple different synapse arrangements (Figure 3A-C). Synapses with clear or dense-cored vesicles on either one side or both sides of the synaptic cleft have been identified (Figure 3A) [64], but the exact function of these uni- and bidirectional synapses remains to be investigated. All ctenophore synapses studied so far are characterized by a presynaptic triad [62] [63] [44] (Figure 3D-F) and this structure can be considered a morphological marker for neuronal cell types. Strikingly, synapses with an unusual localization can be found in a variety of different sensory cells and it appears that any part of a sensory cell can form a synapse onto another cell (Figure 3C).

In cnidarians, synapses are rather sparse and usually contain fewer and slightly larger vesicles [64]. As described above for ctenophores, dense-cored and clear vesicles on either one side or both sides of the synaptic cleft have been identified in interneurons (Figure 3A). Two-way synapses (bidirectional synapses) were the first to be described in cnidarians [66] and later studies confirmed that these synapses were physiologically nonpolarized and thus conducted equally well in either direction [67]. In addition, dense-core vesicles at two-way interneuronal synapses and at neuromuscular synapses have been labelled with antibodies against the neuropeptides RFamide and RWamide indicating the involvement of neuropeptides in synaptic signalling. Consistent with bidirectional signalling, at least one neuron type in Nematostella lacks segregated axo-dendritic compartments as suggested by the live imaging of vesicle transport [68].

Both cnidarian and ctenophore inter-neuronal and neuromuscular synapses lack recognizable postsynaptic specializations in electron micrographs (although in the cnidarian Chrysaora postsynaptic cisternae in the muscle have been described [64](Figure 3B). However, fluorescently tagged Homer and synaptobrevin (putative markers for post and presynapses) showed contact sites between neurites in Nematostella [68]. Consistent with the role of Homer in postsynaptic sites, another study found Homer puncta in transgenic Nematostella at tentacle-retractor and mesentery-retractor muscles and Homer co-expressed with Shank and iGluRs in muscle-cell transcriptomes [69]. In ctenophores, Homer, PSD95 and Shank are expressed broadly and do not reveal any specific postsynaptic cells. However, several iGluR types show highly cell-type-specific expression, potentially marking cells receiving glutamatergic or glycinergic presynaptic input (Figure $3 \mathrm{H}$ ). 

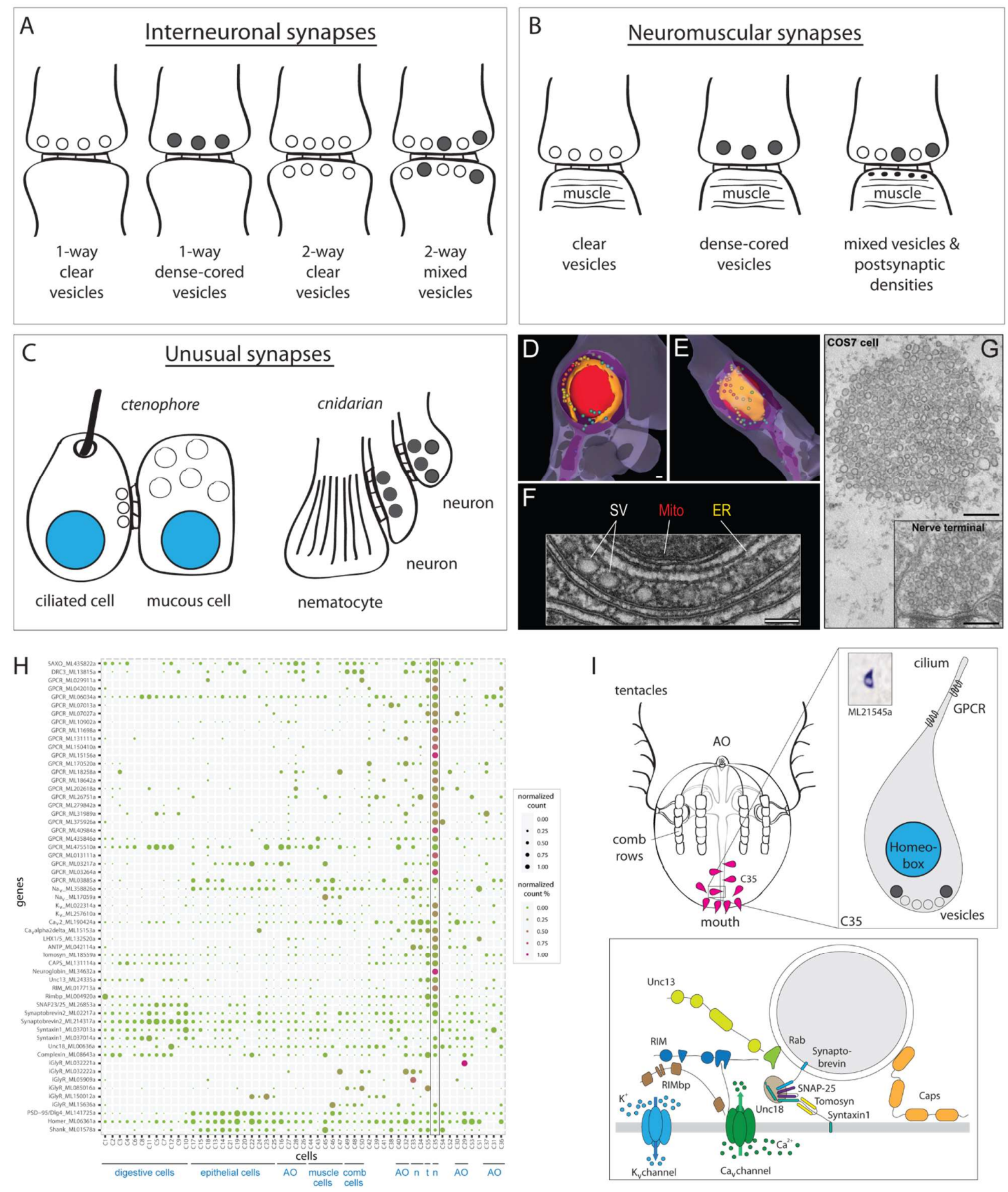

Figure 3: Diversity of synapses in cnidarians and ctenophores, and the ctenophore Unc13-RIM neuron, a potential homolog of bilaterian neurons. (A-B) Schematics of the different synapse arrangements that can be found in both cnidarians and ctenophores (modified from [64]). (C) Unusual synapses in ctenophores and cnidarians. (D-F) 3D reconstruction and electron micrograph of a ctenophore synapse with the characteristic pre-synaptic triad (modified from [44]). (G) The presynaptic proteins synaptophysin and synapsin, when expressed in non-neuronal COS7 cells, induce active zone-like structures (modified from [70]). (H) Single-cell RNA sequencing data of synaptic components, ciliary genes, GPCRs and transcription factors from the ctenophore M. leidyi reveal a ciliated-peptidergic spiking neuron (C35). (I) Drawing of a ctenophore (modified from [71]) 
with the position of the C35 cells highlighted, neuropeptide ML21545a expression pattern and a schematic of a C35 cell. The lower panel shows a putative presynaptic site in the C35 cell. Some components may be involved in the release of both clear and dense core vesicles. Note that M. leidyi RIM lacks a zinc finger domain due to loss (it is present in the sponge Oscarella) [72].

\section{Voltage-gated ion channels and action potentials}

The ability to generate action potentials evolved early in eukaryotes and is thus not restricted to neurons or animals. Action potentials have been detected in green algae [73], diatoms [74] and in a variety of other protists like Stentor, ciliates and heliozoans [75]. In diatoms, monomeric voltage-gated sodium channels [76] with rapid kinetics resembling animal tetrameric $\mathrm{Ca} / \mathrm{Na} / \mathrm{N}$ channels mediate gliding motility. In the green algae Chlamydomonas, action potentials are mediated by voltage dependent $\mathrm{Ca} v$ channels that control the fast switch of flagellar beating $[75,77]$. Thus, Nav/Cav channels evolved early in evolution (likely were present in the last eukaryotic common ancestor) and a picture is emerging that action potentials may have first evolved on flagellar membranes, which is in line with the absence of $\mathrm{Nav} / \mathrm{Ca}_{v}$ channels in eukaryotes without flagella [75]. Action potentials may then have spread from the cilium across the whole cell thus allowing rapid propagation of signals across the cell body [75].

In animals, excitable cells express $K_{v}$ channels, which are responsible for terminating action potentials, alongside $\mathrm{Nav} / \mathrm{Cav}$ channels. The evolutionary history of $\mathrm{K}_{v}$ channels is potentially even older than $\mathrm{Nav} / \mathrm{Ca}_{v}$ channels, as they can be found across protists including choanoflagellates and even in some prokaryotes (archaea Aeropyrum and bacteria Listeria) [78] [79]. Putative $\mathrm{K}_{\mathrm{v}}$ channels are present in all animals, except sponges. A staggering $44 \mathrm{~K}_{\mathrm{v}}$ channels are present in Nematostella, a surprising finding given the rather simple anatomy of its nervous system [80].

The evolutionary origin of action potentials in animals is still unresolved. Previous analyses revealed that $\mathrm{Na} / \mathrm{Ca}$ channels exist in choanoflagellates [81], but it remains unclear what their function is in these organisms. Most sponges lack $\mathrm{Na}_{v}$ channels, however $\mathrm{Ca}_{\mathrm{v}}$ channels and hydrogen channels [26]) are present and action potentials, although slow, have been recorded from glass sponges [82]. Recent electrophysiological experiments in placozoans revealed the presence of fast $\mathrm{Na} v$ dependent action potentials [83] and comparative analyses uncovered a surprising diversity of sodium voltagegated channels in placozoans [83], hinting at the independent expansion of $\mathrm{Na}_{v}$ channels in placozoans. In ctenophores, various voltage-gated ion channels exist. $\mathrm{Ca}_{v}$ channels mediate increases in intraciliary $\mathrm{Ca}^{2+}$ concentration and thus control cilia beating of the comb plate [84]. Two different $\mathrm{Na}_{v}$ channels have been identified in ctenophores, but their function and potential contribution to action potentials has not yet been determined. In cnidarians, sodium-based action potentials have been reported in Nematostella [85], Cyanea [86] and Polyorchis [87]. Calcium action potentials are also very common in non-bilaterians [88] and have likely mediated excitability in the first neurons. Sodium action potentials evolved later independently in placozoans, cnidarians and bilaterians, with unique sodium selectivity filters in each of the three groups [81].

\section{An enigmatic ciliated-peptidergic ctenophore spiking neuron and the homology of (some) synapses}

Genomic comparisons alone have so far not been conclusive about the single or multiple origins of synapses and neurons. The advent of single-cell sequencing has dramatically changed the level of analysis and the insights that can be gained. 
The first single-cell study of the ctenophore $M$. leidyi identified over 50 cell clusters, however, due to the lack of pan-neuronal markers [15], it has initially not been possible to identify neural cells in these data [61]. The recent mapping of prepropeptides to this dataset, combined with tissue stainings, has allowed the identification of several ctenophore neuronal clusters [44]. Building on this development, we have reanalysed the distribution of synaptic and other key molecules in the $M$. leidyi single cell data [61].

To our great surprise, a single cell cluster, C35, stood out. C35 expresses a unique and very interesting combination of genes which allow us to infer its cellular phenotype. It is a ciliated cell likely to have a motile cilium based on the presence of the ciliary markers SAXO [89] and DRC3 [90] (Figure $3 \mathrm{H}$ ) and several axonemal dyneins (not shown). The cell is able to receive specific chemical signals as suggested by the expression of a large number of orphan GPCRs (many of them highly specific to C35). Based on the specific expression of Cav channel subunits and $\mathrm{K}_{v}$ channels, the cell is likely able to generate calcium-mediated action potentials. The cell seems to be specified by a unique homeobox transcription factor code comprising the LIM homeobox transcription factor Lhx1/5 [91] and an ANTP class homeobox transcription factor [92]. C35 also very specifically co-expresses the synaptic active zone factors Unc13 and RIM - previously postulated as key markers of synapse homology [93] - and other presynaptic proteins including Rimbp, SNAP23/25 and a synaptobrevin, suggesting the presence of bilaterian-like small molecule synapses. C35 also specifically expresses at least seven neuropeptides (Figure $3 \mathrm{H}$ ) and the calcium-regulated dense-core-vesicle secretion factor CAPS, indicating that it releases peptidergic vesicles by a canonical pathway. A neuroglobin, related to the bilaterian pan-neuronal neuroglobins [94], is also very specifically expressed in C35. Based on the highly specific neuropeptide markers this cell has been localised to the mouth and pharynx region of Mnemiopsis [44].

Clearly, C35 is not the only neuron type in Mnemiopsis. Many other cells have neuronal morphology, express neuropeptides or have morphologically identified synapses. There are also several synaptic proteins that are expressed more broadly, including Unc18, synaptobrevin, complexin and syntaxin genes, suggesting the presence of molecularly distinct types of neurons and synapses in ctenophores [17]. This agrees with the high morphological diversity of synapses and neuronal cell types.

We interpret the similarities of the C35 cell to bilaterian neural cells as homology and suggest that the eumetazoan common ancestor already had spiking neurons with Unc13-RIM glutamatergic synapses and CAPS-controlled dense core vesicles. The postsynaptic cells likely expressed iGluR and neuropeptide receptors. The presynaptic cell may have also been ciliated and sensory. Based on the position of C35 in Mnemiopsis we speculate that the first neuron may have localised to the mouth region of the animal receiving food-derived signals and signalling to postsynaptic cells that coordinated mouth and pharynx movements during feeding.

If this homology assignment is correct, it does not necessarily mean that all neuronal cells are homologous. Ctenophores underwent extensive parallel evolution in their neural systems, and for example greatly expanded iGluRs, ASICs and innexins [16]. They may have also invented other types of synapses independent of other animals (Fig.3C). It is interesting to note in this context that in vertebrates the presynaptic proteins synaptophysin and synapsin, when expressed in non-neuronal cells, induce active zone-like structures in non-neuronal cells (Figure 3G) [70]. These findings indicate that it is rather easy to induce synapse-like structures and thus, synapses could have "easily" evolved several times separately in different animal lineages.

\section{The Divide-and-Conquer model for early neuronal cell type evolution}

The patterns of origin and distribution of neuronal components are consistent with what we call the Divide-and-Conquer model for neuronal evolution. This model suggests that in early animals 
intercellular signalling was more democratic with many more cell types involved in intercellular signalling on many different channels. This included the release and reception of peptides, glutamate, glycine, NO and ATP in paracrine cell networks ('chemical brains') and not yet synaptic networks $[19,37]$. As recently formulated by Leonid Moroz and co-workers "ancestral neural systems evolved from genetically heterogeneous populations of polarized secretory cells without canonical synapses and capable of volume (non-synaptic) transmission" [19]. Synapses evolved in stem Eumetazoa in some cell types as clusters of secretory vesicles through the scaffolding of pre-existing vesiclesecretion proteins. Several synapse types may have evolved early on and also later lineagespecifically as suggested by synapse diversity in ctenophores and cnidarians. Nostalgic placozoans lost synapses and reverted to the chemical-brain state.

One spiking cell lineage, which we call the Unc13-RIM lineage, was also part of the ancient eumetazoan cell-type mix. The tethering of a Cav channel to the vesicle release machinery by RIM allowed this cell to couple action potentials and calcium influx to rapid vesicle release. Based on the Mnemiopsis data, we speculate that this cell may have occurred around the mouth and may potentially have specialised in chemosensing and the regulation of feeding. After the separation of ctenophores from Cnidaria+Bilateria the democratic unity of cell types had been divided when the Unc13-RIM faction started to become the dominant spiking neuronal lineage. Due to the rapid coupling of firing and vesicle release the Unc13-RIM neuron-type and its synapses gradually conquered the body and took over the control of effectors. This streamlining may have coincided with the advent of predation, where specialised neurons able to do rapid firing and to trigger rapid responses became a life-anddeath question [95]. Going from chemical brains to spiking brains may have started in the region of the mouth, suggesting a savoury connection between macrophagy, predation and brain evolution.

\section{Acknowledgements}

We thank Mari Bergsvåg for the Mnemiopsis picture, Frédérique Varoqueaux for the Trichoplax picture and Patrick Steinmetz for the Nematostella picture. PB would like to thank the Sars Centre core budget and GJ the Leverhulme Trust (Research Project Grant RPG-2018-392) for funding. For the purpose of open access, the authors have applied a CC BY public copyright licence to any Author Accepted Manuscript version arising from this submission.

\section{Conflict of Interest}

The authors declare that they have no conflicts of interest. 


\section{References}

1. Budd GE, Jensen S: The origin of the animals and a "Savannah" hypothesis for early bilaterian evolution. Biological Reviews 2017, 92:446-473.

2. Kapli $P$, Telford MJ: Topology-dependent asymmetry in systematic errors affects phylogenetic placement of Ctenophora and Xenacoelomorpha. Sci Adv 2020, 6.

3. Laumer CE, Gruber-Vodicka H, Hadfield MG, Pearse VB, Riesgo A, Marioni JC, Giribet G: Support for a clade of Placozoa and Cnidaria in genes with minimal compositional bias. Elife 2018, 7.

4. Burkhardt $\mathrm{P}$, Stegmann CM, Cooper B, Kloepper TH, Imig C, Varoqueaux F, Wahl MC, Fasshauer D: Primordial neurosecretory apparatus identified in the choanoflagellate Monosiga brevicollis. Proc Natl Acad Sci U S A 2011, 108:15264-15269.

5. Göhde R, Naumann B, Laundon D, Imig C, McDonald K, Cooper BH, Varoqueaux F, Fasshauer $D$, Burkhardt $P$ : Choanoflagellates and the ancestry of neurosecretory vesicles. Philos Trans R Soc Lond B Biol Sci 2021, 376:20190759.

6. Budd GE, Mann RP: The dynamics of stem and crown groups. Sci Adv 2020, 6:eaaz1626.

7. Budd G, Mann RP: Survival and selection biases in early animal evolution and a source of systematic overestimation in molecular clocks. Interface Focus 2020, 1020190110.

8. Yin Z, Zhu M, Davidson EH, Bottjer DJ, Zhao F, Tafforeau P: Sponge grade body fossil with cellular resolution dating 60 Myr before the Cambrian. Proc Natl Acad Sci U S A 2015, 112:E1453-60.

9. Bobrovskiy I, Hope JM, Nettersheim BJ, Volkman JK, Hallmann C, Brocks JJ: Algal origin of sponge sterane biomarkers negates the oldest evidence for animals in the rock record. Nat Ecol Evol 2021, 5:165-168.

10. van Maldegem LM, Nettersheim BJ, Leider A, Brocks JJ, Adam P, Schaeffer P, Hallmann C: Geological alteration of Precambrian steroids mimics early animal signatures. Nat Ecol Evol 2021, 5:169-173.

11. Nettersheim BJ, Brocks JJ, Schwelm A, Hope JM, Not F, Lomas M, Schmidt C, Schiebel R, Nowack ECM, De Deckker $P$, et al.: Putative sponge biomarkers in unicellular Rhizaria question an early rise of animals. Nat Ecol Evol 2019, 3:577-581.

12. Botting JP, Muir LA: Early sponge evolution: A review and phylogenetic framework. Palaeoworld 2018, 27:1-29.

13. Zhao Y, Vinther J, Parry LA, Wei F, Green E, Pisani D, Hou X, Edgecombe GD, Cong P: Cambrian Sessile, Suspension Feeding Stem-Group Ctenophores and Evolution of the Comb Jelly Body Plan. Curr Biol 2019, 29:1112-1125.e2.

14. Bobrovskiy I, Hope JM, Ivantsov A, Nettersheim BJ, Hallmann C, Brocks JJ: Ancient steroids establish the Ediacaran fossil as one of the earliest animals. Science 2018, 361:12461249.

15. Moroz LL, Kohn AB: Unbiased View of Synaptic and Neuronal Gene Complement in Ctenophores: Are There Pan-neuronal and Pan-synaptic Genes across Metazoa? Integr Comp Biol 2015, 55:1028-1049.

16. Moroz LL, Kocot KM, Citarella MR, Dosung S, Norekian TP, Povolotskaya IS, Grigorenko AP, Dailey C, Berezikov E, Buckley KM, et al.: The ctenophore genome and the evolutionary origins of neural systems. Nature 2014, 510:109-114. 
17. Arendt D: Elementary nervous systems. Philos Trans R Soc Lond B Biol Sci 2021, 376:20200347.

18. Moroz LL: Convergent evolution of neural systems in ctenophores. J Exp Biol 2015, 218:598-611.

19. Moroz LL, Romanova DY, Kohn AB: Neural versus alternative integrative systems: molecular insights into origins of neurotransmitters. Philos Trans R Soc Lond B Biol Sci 2021, 376:20190762.

20. Romanova DY, Heyland A, Sohn D, Kohn AB, Fasshauer D, Varoqueaux F, Moroz LL: Glycine as a signaling molecule and chemoattractant in Trichoplax (Placozoa): insights into the early evolution of neurotransmitters. Neuroreport 2020, 31:490-497.

21. Yu A, Alberstein R, Thomas A, Zimmet A, Grey R, Mayer ML, Lau AY: Molecular lock regulates binding of glycine to a primitive NMDA receptor. Proc Natl Acad Sci U S A 2016, 113:E6786-E6795.

22. Alberstein R, Grey R, Zimmet A, Simmons DK, Mayer ML: Glycine activated ion channel subunits encoded by ctenophore glutamate receptor genes. Proc Natl Acad Sci U S A 2015, 112:E6048-57.

23. Riesgo A, Farrar N, Windsor PJ, Giribet G, Leys SP: The analysis of eight transcriptomes from all poriferan classes reveals surprising genetic complexity in sponges. Mol Biol Evol 2014, 31:1102-1120.

24. Ellwanger $\mathrm{K}$, Eich $\mathrm{A}$, Nickel $\mathrm{M}$ : GABA and glutamate specifically induce contractions in the sponge Tethya wilhelma. J Comp Physiol A Neuroethol Sens Neural Behav Physiol 2007, 193:1-11.

25. Elliott GRD, Leys SP: Evidence for glutamate, GABA and NO in coordinating behaviour in the sponge, Ephydatia muelleri (Demospongiae, Spongillidae). J Exp Biol 2010, 213:23102321.

26. Francis WR, Eitel M, Vargas S, Adamski M, Haddock SHD, Krebs S, Blum H, Erpenbeck D, Wörheide G: The genome of the contractile demosponge Tethya wilhelma and the evolution of metazoan neural signalling pathways. [date unknown], doi:10.1101/120998.

27. Bauknecht $P$, Jékely $G$ : Ancient coexistence of norepinephrine, tyramine, and octopamine signaling in bilaterians. BMC Biol 2017, 15:6.

28. Takeda N, Svendsen CN: Monoamine concentrations in Hydra magnipapillata. Hydrobiologia 1991, 216-217:549-554.

29. Westfall JA, Elliott SR, MohanKumar PS, Carlin RW: Immunocytochemical evidence for biogenic amines and immunogold labeling of serotonergic synapses in tentacles of Aiptasia pallida (Cnidaria, Anthozoa). Invertebr Biol 2005, 119:370-378.

30. Umbriaco $D$, Anctil M, Descarries L: Serotonin-immunoreactive neurons in the cnidarian Renilla koellikeri. J Comp Neurol 1990, 291:167-178.

31. Roopin M, Levy O: Temporal and histological evaluation of melatonin patterns in a “basal” metazoan. J Pineal Res 2012, 53:259-269.

32. Peres R, Reitzel AM, Passamaneck Y, Afeche SC, Cipolla-Neto J, Marques AC, Martindale $M Q$ : Developmental and light-entrained expression of melatonin and its relationship to the circadian clock in the sea anemone Nematostella vectensis. Evodevo 2014, 5:26.

33. Nath RD, Bedbrook CN, Abrams MJ, Basinger T, Bois JS, Prober DA, Sternberg PW, 
Gradinaru V, Goentoro L: The Jellyfish Cassiopea Exhibits a Sleep-like State. Curr Biol 2017, 27:2984-2990.e3.

34. Feuda R, Hamilton SC, Mclnerney JO, Pisani D: Metazoan opsin evolution reveals a simple route to animal vision. Proc Natl Acad Sci U S A 2012, 109:18868-18872.

35. Carlberg M, Alfredsson K, Nielsen SO, Anderson PAV: Taurine-like Immunoreactivity in the Motor Nerve Net of the Jellyfish Cyanea capillata. Biol Bull 1995, 188:78-82.

36. Anderson PAV, Trapido-Rosenthal HG: Physiological and chemical analysis of neurotransmitter candidates at a fast excitatory synapse in the jellyfish Cyanea capillata (Cnidaria, Scyphozoa). Invertebrate Neuroscience 2009, 9:167-173.

37. Jékely G: The chemical brain hypothesis for the origin of nervous systems. Philos Trans $R$ Soc Lond B Biol Sci 2021, 376:20190761.

38. Moroz LL, Romanova DY, Nikitin MA, Sohn D, Kohn AB, Neveu E, Varoqueaux F, Fasshauer $D$ : The diversification and lineage-specific expansion of nitric oxide signaling in Placozoa: insights in the evolution of gaseous transmission. Sci Rep 2020, 10:13020.

39. Musser JM, Schippers KJ, Nickel M, Mizzon G, Kohn AB, Pape C, Hammel JU, Wolf F, Liang C, Hernández-Plaza A, et al.: Profiling cellular diversity in sponges informs animal cell type and nervous system evolution. Cold Spring Harbor Laboratory 2019, doi:10.1101/758276.

40. Quiroga Artigas $G$, Lapébie $P$, Leclère L, Bauknecht $P$, Uveira J, Chevalier S, Jékely $G$, Momose T, Houliston E: A G protein-coupled receptor mediates neuropeptide-induced oocyte maturation in the jellyfish Clytia. PLoS Biol 2020, 18:e3000614.

41. Nakanishi N, Martindale MQ: CRISPR knockouts reveal an endogenous role for ancient neuropeptides in regulating developmental timing in a sea anemone. Elife 2018, 7.

42. Varoqueaux F, Williams EA, Grandemange S, Truscello L, Kamm K, Schierwater B, Jékely G, Fasshauer D: High Cell Diversity and Complex Peptidergic Signaling Underlie Placozoan Behavior. Curr Biol 2018, 28:3495-3501.e2.

43. Senatore A, Reese TS, Smith CL: Neuropeptidergic integration of behavior in Trichoplax adhaerens, an animal without synapses. Journal of Experimental Biology 2017, 220:33813390.

44. Sachkova MY, Nordmann E-L, Soto-Àngel JJ, Meeda Y, Górski B, Naumann B, Dondorp D, Chatzigeorgiou M, Kittelmann M, Burkhardt P: Neuropeptide repertoire and 3D anatomy of the ctenophore nervous system. Current Biology 2021, doi:10.1016/j.cub.2021.09.005.

45. Nikitin M: Bioinformatic prediction of Trichoplax adhaerens regulatory peptides. Gen Comp Endocrinol 2015, 212:145-155.

46. Jékely G: Global view of the evolution and diversity of metazoan neuropeptide signaling. Proc Natl Acad Sci U S A 2013, 110:8702-8707.

47. Wong YY, Le P, Elkhatib W, Piekut T, Senatore A: Transcriptome profiling of Trichoplax adhaerens highlights its digestive epithelium and a rich set of genes for fast electrogenic and slow neuromodulatory cellular signaling. Research Square 2019, doi:10.21203/rs.2.14504/v1.

48. Koch TL, Grimmelikhuijzen CJP: A comparative genomics study of neuropeptide genes in the cnidarian subclasses Hexacorallia and Ceriantharia. BMC Genomics 2020, 21:666.

49. Takahashi T, Muneoka Y, Lohmann J, Lopez de Haro MS, Solleder G, Bosch TC, David CN, 
Bode HR, Koizumi O, Shimizu H, et al.: Systematic isolation of peptide signal molecules regulating development in hydra: LWamide and PW families. Proc Natl Acad Sci U S A 1997, 94:1241-1246.

50. Elphick MR, Mirabeau O, Larhammar D: Evolution of neuropeptide signalling systems. $J$ Exp Biol 2018, 221.

51. Yañez-Guerra LA, Zhong X, Moghul I, Butts T, Zampronio CG, Jones AM, Mirabeau O, Elphick MR: Echinoderms provide missing link in the evolution of PrRP/sNPF-type neuropeptide signalling. Elife 2020, 9.

52. Mirabeau O, Joly J-S: Molecular evolution of peptidergic signaling systems in bilaterians. Proc Natl Acad Sci U S A 2013, 110:E2028-37.

53. Kumar D, Strenkert D, Patel-King RS, Leonard MT, Merchant SS, Mains RE, King SM, Eipper BA: A bioactive peptide amidating enzyme is required for ciliogenesis. Elife 2017, 6.

54. Luxmi R, Kumar D, Mains RE, King SM, Eipper BA: Cilia-based peptidergic signaling. PLoS Biol 2019, 17:e3000566.

55. de Pavans Ceccatty M: Les bases ultrastructurales des communications intercellulaires dans les oscules de quelques éponges. In Symp. Zool. Soc. Lond. . 1970:449-466.

56. Lentz TL: Histochemical localization of neurohumors in a sponge. J Exp Zool 1966, 162:171-179.

57. Assmann M, Kuhn A, Dürrnagel S, Holstein TW, Gründer S: The comprehensive analysis of DEG/ENaC subunits in Hydra reveals a large variety of peptide-gated channels, potentially involved in neuromuscular transmission. BMC Biol 2014, 12:84.

58. Zang $\mathrm{H}$, Nakanishi $\mathrm{N}$ : Expression Analysis of Cnidarian-Specific Neuropeptides in a Sea Anemone Unveils an Apical-Organ-Associated Nerve Net That Disintegrates at Metamorphosis. Front Endocrinol 2020, 11:63.

59. Quiroga Artigas G, Lapébie P, Leclère L, Bauknecht P, Uveira J, Chevalier S, Jékely G, Momose $T$, Houliston $E$ : A G protein-coupled receptor mediates neuropeptide-induced oocyte maturation in the jellyfish Clytia. PLoS Biol 2020, 18:e3000614.

60. Sebé-Pedrós A, Saudemont B, Chomsky E, Plessier F, Mailhé M-P, Renno J, Loe-Mie Y, Lifshitz A, Mukamel Z, Schmutz S, et al.: Cnidarian Cell Type Diversity and Regulation Revealed by Whole-Organism Single-Cell RNA-Seq. Cell 2018, 173:1520-1534.e20.

61. Sebé-Pedrós A, Chomsky E, Pang K, Lara-Astiaso D, Gaiti F, Mukamel Z, Amit I, Hejnol A, Degnan BM, Tanay A: Early metazoan cell type diversity and the evolution of multicellular gene regulation. Nat Ecol Evol 2018, 2:1176-1188.

62. Horrige GA, Mackay B: Neurociliary Synapses in Pleurobrachia (Ctenophora). J Cell Sci 1964, s3-105:163-174.

63. Hernandez-Nicaise ML: Specialized connexions between nerve cells and mesenchymal cells in ctenophores. Nature 1968, 217:1075-1076.

64. Westfall IA: Ultrastructure of synapses in the first-evolved nervous systems. J Neurocytol 1996, 25:735-746.

65. Arendt D: The Evolutionary Assembly of Neuronal Machinery. Curr Biol 2020, 30:R603R616.

66. Horridge GA, Mackay B: Naked Axons and Symmetrical Synapses in Coelenterates. J Cell 
Sci 1962, s3-103:531-541.

67. Anderson PA: Physiology of a bidirectional, excitatory, chemical synapse. J Neurophysiol 1985, 53:821-835.

68. Stone MC, Kothe GO, Rolls MM, Jegla T: Cytoskeletal and synaptic polarity of LWamidelike+ ganglion neurons in the sea anemone. J Exp Biol 2020, 223.

69. Cole AG, Kaul S, Jahnel SM, Steger J, Zimmerman B: Muscle cell type diversification facilitated by extensive gene duplications. bioRxiv.

70. Park D, Wu Y, Lee S-E, Kim G, Jeong S, Milovanovic D, Camilli PD, Chang S: Cooperative function of synaptophysin and synapsin in the generation of synaptic vesicle-like clusters in non-neuronal cells. Nat Commun 2021, 12:263.

71. Fischer $\mathrm{AH}$, Pang K, Henry JQ, Martindale MQ: A cleavage clock regulates features of lineage-specific differentiation in the development of a basal branching metazoan, the ctenophore Mnemiopsis leidyi. Evodevo 2014, 5:4.

72. Piekut T, Wong YY, Walker SE, Smith CL, Gauberg J, Harracksingh AN, Lowden C, Novogradac BB, Cheng H-YM, Spencer GE, et al.: Early Metazoan Origin and Multiple Losses of a Novel Clade of RIM Presynaptic Calcium Channel Scaffolding Protein Homologs. Genome Biol Evol 2020, 12:1217-1239.

73. Hope AB, Findlay GP: THE ACTION POTENTIAL IN CHARA. Plant Cell Physiol 1964, 5:377379.

74. Taylor AR: A fast $\mathbf{N a + / C a 2 + - b a s e d ~ a c t i o n ~ p o t e n t i a l ~ i n ~ a ~ m a r i n e ~ d i a t o m . ~ P L o S ~ O n e ~ 2 0 0 9 , ~}$ 4:e4966.

75. Brunet $T$, Arendt $D$ : From damage response to action potentials: early evolution of neural and contractile modules in stem eukaryotes. Philos Trans R Soc Lond B Biol Sci 2016, 371:20150043.

76. Helliwell KE, Chrachri A, Koester JA, Wharam S, Verret F, Taylor AR, Wheeler GL, Brownlee $\mathrm{C}$ : Alternative Mechanisms for Fast $\mathrm{Na}+/ \mathrm{Ca} 2+$ Signaling in Eukaryotes via a Novel Class of Single-Domain Voltage-Gated Channels. Curr Biol 2019, 29:1503-1511.e6.

77. Harz H, Hegemann P: Rhodopsin-regulated calcium currents in Chlamydomonas. Nature 1991, 351:489-491.

78. Jiang Y, Lee A, Chen J, Ruta V, Cadene M, Chait BT, MacKinnon R: X-ray structure of a voltage-dependent K+ channel. Nature 2003, 423:33-41.

79. Santos JS, Lundby A, Zazueta C, Montal M: Molecular template for a voltage sensor in a novel $\mathrm{K}+$ channel. I. Identification and functional characterization of $\mathrm{KvLm}$, a voltagegated K+ channel from Listeria monocytogenes. J Gen Physiol 2006, 128:283-292.

80. Moran $\mathrm{Y}$, Barzilai MG, Liebeskind $\mathrm{BJ}$, Zakon $\mathrm{HH}$ : Evolution of voltage-gated ion channels at the emergence of Metazoa. J Exp Biol 2015, 218:515-525.

81. Liebeskind $\mathrm{BJ}$, Hillis DM, Zakon $\mathrm{HH}$ : Evolution of sodium channels predates the origin of nervous systems in animals. Proc Natl Acad Sci U S A 2011, 108:9154-9159.

82. Leys SP, Mackie GO: Electrical recording from a glass sponge. Nature 1997, 387:29-30.

83. Romanova DY, Smirnov IV, Nikitin MA, Kohn AB, Borman Al, Malyshev AY, Balaban PM, Moroz LL: Sodium action potentials in placozoa: Insights into behavioral integration and evolution of nerveless animals. Biochem Biophys Res Commun 2020, 532:120-126. 
84. Moss AG, Tamm SL: A calcium regenerative potential controlling ciliary reversal is propagated along the length of ctenophore comb plates. Proc Natl Acad Sci U S A 1987, 84:6476-6480.

85. Gur Barzilai M, Reitzel AM, Kraus JEM, Gordon D, Technau U, Gurevitz M, Moran Y: Convergent evolution of sodium ion selectivity in metazoan neuronal signaling. Cell Rep 2012, 2:242-248.

86. Anderson PA, Schwab WE: Action potential in neurons of motor nerve net of Cyanea (Coelenterata). J Neurophysiol 1983, 50:671-683.

87. Spencer AN, Satterlie RA: The action potential and contraction in subumbrellar swimming muscle ofPolyorchis penicillatus (Hydromedusae). J Comp Physiol 1981, 144:401-407.

88. Jaffe LF: The propagation speeds of calcium action potentials are remarkably invariant. Biol Cell 2003, 95:343-355.

89. Dacheux D, Roger B, Bosc C, Landrein N, Roche E, Chansel L, Trian T, Andrieux A, Papaxanthos-Roche A, Marthan R, et al.: Human FAM154A (SAXO1) is a microtubulestabilizing protein specific to cilia and related structures. J Cell Sci 2015, 128:1294-1307.

90. Awata J, Song K, Lin J, King SM, Sanderson MJ, Nicastro D, Witman GB: DRC3 connects the $\mathrm{N}-\mathrm{DRC}$ to dynein $\mathrm{g}$ to regulate flagellar waveform. Mol Biol Cell 2015, 26:2788-2800.

91. Simmons DK, Pang K, Martindale MQ: Lim homeobox genes in the Ctenophore Mnemiopsis leidyi: the evolution of neural cell type specification. Evodevo 2012, 3:1-11.

92. Ryan JF, Pang K, NISC Comparative Sequencing Program, Mullikin JC, Martindale MQ, Baxevanis AD: The homeodomain complement of the ctenophore Mnemiopsis leidyi suggests that Ctenophora and Porifera diverged prior to the ParaHoxozoa. Evodevo 2010, 1:9.

93. Marlow $\mathrm{H}$, Arendt $\mathrm{D}$ : Evolution: ctenophore genomes and the origin of neurons. Curr Biol 2014, 24:R757-61.

94. Lechauve C, Jager M, Laguerre L, Kiger L, Correc G, Leroux C, Vinogradov S, Czjzek M, Marden MC, Bailly X: Neuroglobins, Pivotal Proteins Associated with Emerging Neural Systems and Precursors of Metazoan Globin Diversity. Journal of Biological Chemistry 2013, 288:6957-6967.

95. Monk T, Paulin MG: Predation and the Origin of Neurones. Brain, Behavior and Evolution 2014, 84:246-261. 


\section{Annotated subset of particularly important papers:}

${ }^{* *}$ Stone, Michelle C., Gregory O. Kothe, Melissa M. Rolls, and Timothy Jegla. 2020.

"Cytoskeletal and Synaptic Polarity of LWamide-Like+ Ganglion Neurons in the Sea Anemone."

The Journal of Experimental Biology 223 (Pt 21). https://doi.org/10.1242/jeb.233197.

This paper uses transgenesis and live imaging in the sea anemone Nematostella vectensis. The authors demonstrate that in one neuron type all neurites have axon-like microtubule polarity and they could observe the trafficking of both presynaptic and postsynaptic markers.

**Sebé-Pedrós, Arnau, Elad Chomsky, Kevin Pang, David Lara-Astiaso, Federico Gaiti, Zohar Mukamel, Ido Amit, Andreas Hejnol, Bernard M. Degnan, and Amos Tanay. 2018. "Early Metazoan Cell Type Diversity and the Evolution of Multicellular Gene Regulation." Nature Ecology \& Evolution 2 (7): 1176-88.

This paper reports single-cell sequencing data from the sponge Amphimedon queenslandica, the ctenophore $M$. leidyi and the placozoan $T$. adhaerens. The authors report the cell-type diversity and transcription-factor regulatory programmes for these non-bilaterians.

${ }^{*}$ Romanova DY, Smirnov IV, Nikitin MA, Kohn AB, Borman AI, Malyshev AY, Balaban PM, Moroz LL: Sodium action potentials in placozoa: Insights into behavioral integration and evolution of nerveless animals. Biochem Biophys Res Commun 2020, 532:120-126.

Here the authors demonstrate the presence of sodium-dependent action potentials in placozoans. They also analyse the evolution of $\mathrm{Na} v$ channels and uncover an independent evolutionary radiation of voltage gated sodium channels in these animals.

*Park, Daehun, Yumei Wu, Sang-Eun Lee, Goeun Kim, Seonyoung Jeong, Dragomir Milovanovic, Pietro De Camilli, and Sunghoe Chang. 2021. "Cooperative Function of Synaptophysin and Synapsin in the Generation of Synaptic Vesicle-like Clusters in NonNeuronal Cells." Nature Communications 12 (1): 263.

This paper reports that synapsin, when expressed together with the synaptic-vesicle membrane protein synaptophysin in fibroblasts, can organise vesicle clusters similar to synaptic-vesicle clusters. This minimal system also has implications for understanding the evolution of synapses.

**Luxmi, Raj, Dhivya Kumar, Richard E. Mains, Stephen M. King, and Betty A. Eipper. 2019.

“Cilia-Based Peptidergic Signaling." PLoS Biology 17 (12): e3000566.

Here the authors report the presence of PAM and amidated peptides in ciliary ectosomes from the green alga Chlamydomonas. They show that one amidated peptide acts as a chemoattractant. This beautiful work uncovers a deep ancestry for metazoan neuropeptide signalling.

**Kapli, Paschalia, and Maximilian J. Telford. 2020. "Topology-Dependent Asymmetry in Systematic Errors Affects Phylogenetic Placement of Ctenophora and Xenacoelomorpha." Science Advances 6 (50). https://doi.org/10.1126/sciadv.abc5162.

In this paper the authors use simulations and empirical data to show that the Ctenophora-first but not the Porifera-first topology can be explained by systematic errors. Porifera-first can only be 
explained by real phylogenetic signal. This large asymmetry in systematic bias strongly supports Porifera-first. 